\title{
A Rare Association: Apical Hypertrophic Cardiomyopathy with Multiple Coronary Artery-Left Ventricular Fistulae
}

\author{
Isabel Monedero-Sánchez, MD, Pablo Robles-Velasco, MD, Amador Rubio-Caballero, \\ MD, Yago González-Doforno, MD, Cecilia Marco-Quirós, MD, Victoria Espejo-Bares, MD, \\ Verónica Artiaga-de-Ia-Barrera, MD and Carla Jiménez-Martínez, MD
}

Department of Cardiology, Hospital Universitario Fundación Alcorcón, Spain

*Corresponding author: Isabel Monedero-Sánchez, Departament of Cardiology, Hospital Universitario Fundación Alcorcón, Calle Budapest N 1, 28922, Alcorcón (Madrid), Spain, Tel: +34-699-29-09-63, Fax: +34-916219890

\begin{abstract}
A 59-year-old woman was referred for evaluation of chest pain and she was diagnosed of acute coronary syndrome. Coronary arteriography showed a severe stenosis of left anterior descending artery but also the presence of multiple coronary artery-left ventricle micro-fistulae. Echocardiographic findings were consistent with apical hypertrophic cardiomyopathy and the fistulae were also patent by colour Doppler echocardiography. Little is known about clinical features of micro-fistulae arising from both coronary arteries and emptying into left ventricle, especially when they are associated with apical hypertrophic cardiomyopathy.
\end{abstract}

\section{Keywords}

Coronary fistulae, Hypertrophic cardiomyopathy

\section{Learning Objective}

We describe a case of apical hypertrophic cardiomyopathy associated with multiple fistulae originating from both left and right coronary artery and draining into the left ventricle. The co-existence of both entities is very infrequent and has been rarely reported. Additionally, the presence of concomitant atherosclerotic coronary artery disease, as in this case, is exceptional.

\section{Introduction}

Coronary artery fistulae are an uncommon finding of coronary angiographies. Frequently the fistulae arise from the right coronary artery and drain into the right heart chambers. Their co-existence with apical hypertrophic cardiomyopathy is very rare and the pathophysiologic mechanisms involved in this association are widely unknown.

We present a case of multiple fistulae originating from both coronary arteries and draining into the left ventricle associated with apical hypertrophic cardiomyopathy, presenting as acute coronary syndrome, which was diagnosed by coronary arteriography and colour Doppler echocardiography.

\section{Case Description}

A 59-year-old woman was admitted to our hospital for chest pain. She was smoker, with no previous history of cardiovascular disease. Her electrocardiogram showed sinus rhythm with left ventricular hypertrophy criteria and significant ST segment depression in leads V2 to V5, and cardiac troponin levels were elevated.

Transthoracic echocardiography showed diffuse hypertrophy of the mid to apical left ventricular walls (Figure 1A). Colour Doppler echocardiography revealed multiple linear colour-flow signals perpendicular to the epicardium inside the hypertrophic segments (white arrows in Figure 1B). A diastolic flow pattern, suggestive of coronary flow, was identified at the colour signals by pulse-wave Doppler (white stars in Figure 1C).

After medical treatment she underwent cardiac catheterization within the first 24 hours from the onset of symptoms. It showed diffuse non-obstructive 


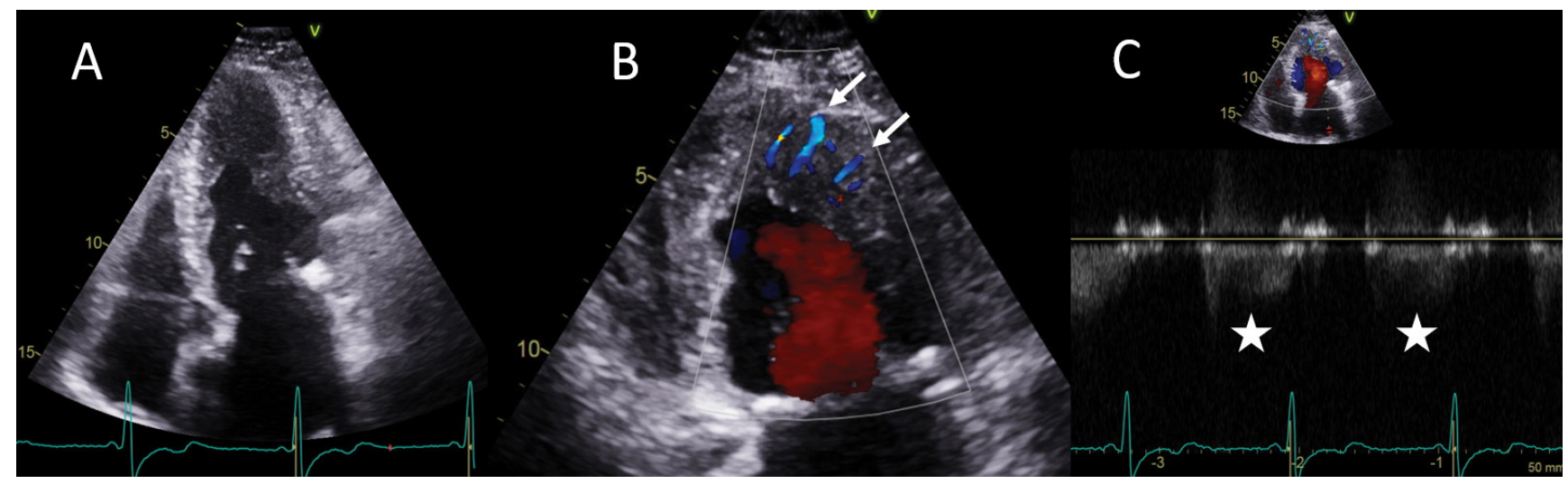

Figure 1: A) Transthoracic echocardiography showed diffuse hypertrophy of the mid to apical left ventricular walls, suggestive of apical hypertrophic cardiomyopathy; B) Colour Doppler revealed multiple linear colour-flow signals (arrows) inside the hypertrophic segments; C) Pulse-wave Doppler identified a diastolic flow pattern, suggestive of coronary flow, at the colour signals (stars), corresponding with coronary fistulae.

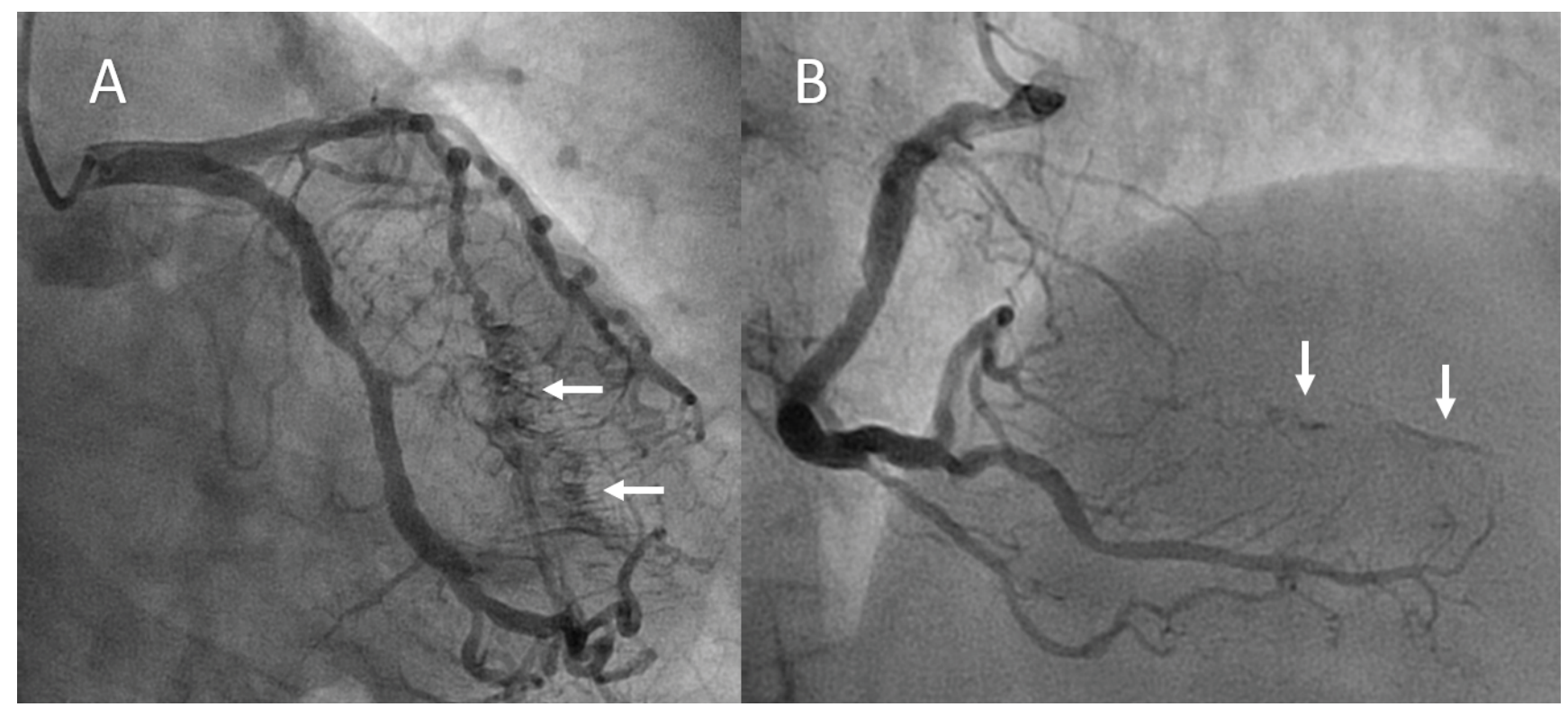

Figure 2: Coronary arteriography revealed the presence of multiple fistulae (arrows) originating from both the left coronary artery system A) and the right coronary artery; B) and draining into the left ventricular cavity.

coronary lesions and severe stenosis of mid segment of left anterior descending artery, which was treated with stent implantation. Coronary arteriography also revealed the presence of multiple fistulae originating from both the left coronary artery system and the right coronary artery and draining into the left ventricular cavity (white arrows in Figure 2A and Figure 2B).

Although echocardiographic findings could initially suggest hypertrabeculation of the endocardial side mimicking left ventricular noncompaction, coronary arteriography demonstrated that linear colour-flow signals inside the hypertrophic segments were correlated with numerous coronary fistulae draining into the left ventricle, and noncompaction of the myocardium was reasonably ruled out. So finally the diagnosis was consistent with apical hypertrophic cardiomyopathy associated with multiple coronary artery-left ventricle microfistulae.

\section{Discussion}

Coronary artery fistulas are defined as single or multiple communications between one or more coronary arteries and one of the four cardiac chambers or other vessels as pulmonary artery. They have been reported in $\approx 0.2 \%$ of coronary angiographies. They usually have a congenital etiology and, in $20 \%$ of the cases, they are associated with other cardiac abnormalities as aortic and pulmonary atresia and patent ductus arteriosus [1].

In general terms, coronary fistulae frequently originate from right coronary artery and drain into right heart chambers. Clinical impact can vary from asymptomatic patients to symptoms of angina pectoris, myocardial infarction, heart failure, ventricular arrhythmias, syncope and sudden death, mainly due to myocardial ischemia because of coronary steal phenomenon [2]. 
The association between coronary fistulae and apical hypertrophic cardiomyopathy is very rare. In the few cases reported in the literature in this scenario, most of the fistulae drain from the right and left coronary arteries to the left ventricle [3]. Some studies suggest that this phenotype might be explained as the result of an embryonic development anomaly. Under normal circumstances foetal myocardial sinusoids, that arise from endothelial protrusions into the intertrabecular spaces, experience significant regression with the development, resulting in the formation of Thebesian vessels of the adult heart. Interference with developmental changes might produce a partial persistence of embryonic myocardial sinusoids and an abnormally prominent Thebesian system, which would correlate with the appearance of multiple coronary microfistulae [4].

When coronary fistulae and apical hypertrophic cardiomyopathy coexist, it is not clear if the apical hypertrophy could be a reactive change to chronic volume overload due to the coronary artery to left ventricular shunt, or if the disarray of myocardial cells in hypertrophic segments facilitates the development of multiple coronary microfistulae [4].

The presence of concomitant atherosclerotic coronary artery disease is exceptional. In our case, it was probably favored by the smoking habit of the patient. It remains unclear if there is an association with premature atherosclerosis.

The diagnosis is usually difficult because symptoms, clinical signs, laboratory test results and ECG findings are nonspecific. In most cases, coronary fistulae are identified during invasive coronary angiography or computed tomography coronary angiographyperformed to evaluate patients with chest pain. Colour Doppler echocardiography can demonstrate linear diastolic flow signals inside the myocardium corresponding with the presence of these fistulas.

Treatment of coronary fistulae is essentially medical (including $\beta$-blockers, calcium channel blockers, nitrates and ivabradine). Only in the presence of substantial significant shunts or complications (including progressive enlargement or rupture, bacterial endocarditis or significant pulmonary hypertension) patients may undergo percutaneous occlusion or surgical ligation [5].

\section{Authors Contribution}

All authors have contributed equally.

\section{Conflicts of Interest}

The authors declare that there is no conflict of interest.

\section{References}

1. Yamanaka O, Hobbs RE (1990) Coronary artery anomalies in 126,595 patients undergoing coronary arteriography. Cathet Cardiovasc Diagn 21: 28-40.

2. Meena DS, Meena CB, Parvez J (2017) Hypertrophic obstructive cardiomyopathy with multiple coronary arteries to right ventricular microfistulas: A case report and review of the literatura. J Med Case Rep 11: 24.

3. Dresios C, Apostolakis S, Tzortzis S, Lazaridis K, Gardikiotis A (2010) Apical hypertrophic cardiomyopathy associated with multiple coronary artery-left ventricular fistulae: A report of a case and review of the literature. Eur J Echocardiogr 11: E9.

4. Hong GR, Choi SH, Kang SM, Lee MH, Rim SJ, et al. (2003) Multiple coronary artery-left ventricular microfistulae in a patient with apical hypertrophic cardiomyopathy: A demonstration by transthoracic color Doppler echocardiography. Yonsei Med J 44: 710-714.

5. Said SA, Schiphorst RH, Derksen R, Wagenaar L (2013) Coronary-cameral fistulas in adults. World J Cardiol 5: 329336. 